Quim. Nova, Vol. 34, No. 3, 424-429, 2011

\title{
ESTUDO DA INFLUÊNCIA DOS PARTICULADOS NO FENÔMENO DE AGREGAÇÃO DOS ASFALTENOS POR ESPECTROMETRIA DE VARREDURA ÓPTICA
}

\author{
Claudete Bernardo Henriques* \\ Otimização/Qualidade de Produtos, Refinaria de Paulínia, PETROBRAS, Paulínia - SP / Departamento de Processos Químicos, \\ Faculdade de Engenharia Química, Universidade Estadual de Campinas, CP 6066, 13083-970 Campinas - SP / Departamento de \\ Química Analítica, Instituto de Química, Universidade Estadual de Campinas, CP 6154, 13084-971 Campinas - SP, Brasil \\ Alessandra Winter, Erika Tomie Koroishi e Rubens Maciel Filho \\ Departamento de Processos Químicos, Faculdade de Engenharia Química, Universidade Estadual de Campinas, CP 6066, \\ 13083-970 Campinas - SP, Brasil \\ Maria Izabel Maretti Silveira Bueno \\ Departamento de Química Analítica, Instituto de Química, Universidade Estadual de Campinas, CP 6154, 13084-971 Campinas \\ - SP, Brasil
}

Recebido em 27/5/10; aceito em 15/10/10; publicado na web em 31/1/11

\begin{abstract}
STUDY OF THE INFLUENCE OF THE PARTICULATE IN AGGREGATION PHENOMENON OF THE ASPHALTENES BY OPTICAL SCANNING SPECTROMETRY. The processing of national petroleums causes many operational problems due to the asphaltene aggregation phenomena. To evaluate the behavior of these phenomena, a study based on optical scan technique was carried out to understand the behavior of pure oils and their mixtures. The evaluation according to criteria established by ASTM D7061-06 ${ }^{1}$ gives a result of asphaltene flocculation of according to the separability number. In this study, the aspects related to the existence of colloidal forms of peptized asphaltenes in distinct solvents present in petroleums can cause changes in the kinetics of asphaltene flocculation.
\end{abstract}

Keywords: asphaltenes; aggregation phenomena; separability number.

\section{INTRODUÇÃO}

O termo "fractal" foi introduzido na década de 70 pelo matemático Benôit Mandelbrot para designar objetos e estruturas complexas dotadas da propriedade de autossimilaridade. Estruturas autossimilares possuem detalhes (ramificações, poros ou rugosidades) em certa faixa de escala de comprimento, cuja forma é a mesma (estatisticamente) em cada escala de observação nessa faixa. Assim, se uma parte da estrutura for ampliada terá a mesma forma do todo. Várias investigações já foram feitas em relação à natureza fractal da agregação dos asfaltenos. Janardhan e Mansoori avaliaram diversos modelos, com intuito de se estabelecer experimentalmente uma evidência sobre esta característica dos asfaltenos. ${ }^{2}$

A natureza fractal dos agregados de asfaltenos foi descrita a partir de dois modelos: modelo termodinâmico contínuo e modelo estérico coloidal. No modelo termodinâmico contínuo, assume-se que as moléculas de asfaltenos encontram-se dissolvidas no óleo, na forma de um polímero polidisperso heterogêneo e dependem da massa molecular dos asfaltenos. Este modelo assume também que ambas as fases líquidas e sólidas são iguais para toda a fração e que a fase sólida está livre do solvente. O modelo estérico coloidal está fundamentado no princípio de que os asfaltenos estão suspensos no óleo sob a forma de partículas sólidas. Esta suspensão existe devido à presença das resinas, e é mantida devido às forças eletrostáticas de repulsão entre as moléculas de resina absorvidas nas superfícies. A condição necessária para que ocorra o início da floculação é que a concentração das resinas esteja abaixo de um valor crítico e este valor é calculado através da equação do potencial químico das moléculas de resinas na fase líquida e sólida. ${ }^{2}$

\footnotetext{
*e-mail: claudete.b.h@petrobras.com.br
}

No modelo coloidal, acredita-se que os asfaltenos se agregam sob a forma de micelas ou micelas reversas. As micelas são formadas a partir de uma determinada concentração na solução, denominada concentração micelar crítica (CMC). ${ }^{3}$

Oyekunle estudou os efeitos que controlam a estabilização das micelas de asfaltenos. Para isto, determinou o tamanho das partículas de asfaltenos (4 a $10 \mathrm{~nm}$ ) e de suas micelas (15 a $30 \mathrm{~nm}$ ). Dois parâmetros chaves controlam esta estabilidade. O primeiro é a relação dos compostos aromáticos e saturados e o segundo é a relação entre as resinas e os asfaltenos. Quando estas relações diminuem, as micelas de asfaltenos coalescerão, formando agregados maiores. Estas duas relações foram utilizadas neste estudo para descrever a relação entre a composição química dos grupos funcionais e as propriedades físicas dos asfaltenos e são expressas através de dois índices: o índice de asfaltenos (Ia) e o índice Gaestel (Ic). ${ }^{4}$

As resinas têm papel fundamental na estabilidade dos asfaltenos em solução, dispersando os asfaltenos e impedindo, assim, a sua separação. O poder de solubilização das resinas é afetado pelo grau de aromaticidade das moléculas, sendo que o índice Gaestel estabelece a capacidade de dispersão dos maltenos nos asfaltenos. Quando o Ic aumenta, ocorre a diminuição da estabilidade coloidal, sendo um importante parâmetro para análise de diferentes amostras de asfaltos.

A razão entre resinas e asfaltenos nos estudos de suas influências sobre a estabilidade de emulsões de petróleos indicou que a diminuição desta razão aumenta a estabilidade, sendo independente de suas origens. ${ }^{3}$

Vários autores atribuíram a presença de asfaltenos como uma das principais razões para a formação de depósito de coque em permutadores e fornos. Esses autores consideram que a existência da forma coloidal dos asfaltenos pepitizados pelas resinas e outros 
solventes presentes no petróleo possam ser a causa da formação destes depósitos associada a mudanças de pressão, temperatura e composição dos solventes. ${ }^{5-8}$

Kilpatrick e McLean estudaram o efeito do fenômeno de agregação dos asfaltenos em uma mistura de solvente (7:3 heptano:tolueno) e a estabilidade de uma emulsão água em óleo com quatro tipos de petróleos diferentes. Concluiu-se que a relação resina/asfalteno dos petróleos estudados foi mais influente no processo de estabilização da emulsão: isso foi atribuído ao caráter polar e/ou aromático das resinas presentes nesses óleos. Portanto, alguma mudança na aromaticidade, na relação resina/asfaltenos e/ou presença de um grupo funcional polar resultam na desestabilização e produção de emulsão. O decréscimo de um destes parâmetros ou a combinação dos mesmos resulta em uma rápida precipitação dos asfaltenos. ${ }^{9}$

A determinação de estabilidade de óleos é usualmente feita através dos métodos spot test (teste da mancha), hot filtration (precipitação em n-heptano) e $P$-value (correlaciona o produto com a solubilidade do n-heptano). Estes métodos avaliam a compatibilidade dos óleos, apenas levando em conta o comportamento dos asfaltenos na estabilidade das emulsões. ${ }^{10,11}$

Neste trabalho, a técnica de varredura ótica foi utilizada para avaliar a estabilidade de dois conjuntos de dados formados por petróleos puros e suas misturas. Já é de conhecimento que agentes emulsificantes, como os asfaltenos, agregados de resinas/asfaltenos e partículas sólidas adsorvem na interface das gotas de água devido à afinidade das moléculas, sendo que as extremidades hidrofílicas ficam alinhadas com a água, enquanto as lipofílicas ficam alinhadas com o óleo, formando uma película orientada e estabilizada. Sendo assim, é necessário um estudo conjunto destes fenômenos, o que é possível através da técnica proposta.

\section{CONSIDERAÇÕES SOBRE PRECIPITAÇÃO DOS ASFALTENOS}

De maneira simples, podemos expressar a solubilidade dos asfaltenos no petróleo através da teoria apresentada no estudo de Hidelbrand e Scatchard, onde se estabelece uma relação entre a energia livre de mistura de dois componentes puros a partir da entropia de mistura (considerada ideal). Para o petróleo são consideradas as seguintes aproximações: o petróleo é considerado como solvente; os asfaltenos são considerados soluto; os asfaltenos são componentes únicos do sistema; o sistema é considerado como um sistema com apenas dois componentes e, a concentração dos asfaltenos deve ser considerada ínfima (>>diluídos).

A precipitação dos asfaltenos, de acordo com essa teoria, induzida pelo solvente descreve que, quando a razão entre os volumes molares diminui, a diferença entre os parâmetros de solubilidade aumenta, reduzindo a solubilidade dos asfaltenos, o que resulta na sua precipitação.

A precipitação dos asfaltenos foi descrita por Hirschberg. A adição de solventes alifáticos (n-pentano, n-heptano, etc) reduz o parâmetro de solubilidade do meio até o ponto critico, no qual o poder de solvência se torna insuficiente para manter as frações polares (asfaltenos, asfaltenos/resinas) em solução, promovendo a precipitação. Este parâmetro estabelece uma clara correlação entre os processos de separação dos asfaltenos induzida pela adição de solventes alifáticos (n-heptano). A aplicação de parâmetros de solubilidade do óleo e de compatibilidade entre petróleos para descrever fenômenos de agregação leva em consideração: parâmetros de solubilidade $(\delta)$, que por sua vez correlacionam calor da mistura $(\Delta \mathrm{E})$ e volumes molares do meio solvente (v). Quando volumes molares são muito diferentes, o parâmetro de solubilidade ( $\delta$ ) é considerado a somatória entre as frações volumétricas de cada componente $\left(\varphi_{\mathrm{i}}\right)$ e do seu parâmetro de solubilidade de cada componente $\left(\delta_{\mathrm{i}}\right)$. Esta relação é aplicada a sistemas em modelos que analisam a precipitação dos asfaltenos que foram diluídos em tolueno e titulados em n-heptano, sempre que estes estiveram em concentração muito diluídas.

Esta correlação tem sido muito utilizada para estabelecer o parâmetro de solubilidade e o ponto de viragem na precipitação dos asfaltenos utilizando NIR. Utilizam também valores fixos para esta precipitação: $\delta$ viragem 16,4 e $16,6 \mathrm{MPa}^{1 / 2}$, obtidas a partir de diferentes soluções de asfaltenos, com petróleos de origens diversas.

Os autores concluíram que o parâmetro de solubilidade dos asfaltenos independe de sua origem ou concentração, com comportamento similar ao descrito teoricamente pelas macromoléculas (teoria de Hildebrand-Setchard e Hansen).

Se considerarmos um petróleo como componente único em um processo de titulação para determinar a sua precipitação em n-heptano, o sistema seria formado pelo óleo (pseudo-componente único), independente de sua concentração e composição; o parâmetro de solubilidade para diversas amostras de petróleo mediante a titulação com n-heptano é descrito pela Equação 1:

$$
\delta_{\text {prec }}=\delta_{o} \varphi_{o}+\delta_{h} \varphi_{h}
$$

*os asfaltenos encontram-se em concentrações muito baixas (dois componentes do sistema)

A aplicação do NIR para detecção do ponto de precipitação é prevista no momento da redução da absorbância e a partir do momento em que começam a aparecer partículas no meio. Neste ponto, começa o acréscimo da absorbância devido à dispersão das partículas no meio. O resultado é dado em função do volume de n-heptano, correspondente ao ponto em que a curva de titulação apresenta um mínimo.

A aplicação do UV-Vis para detecção do ponto de precipitação dos asfaltenos ocorre da seguinte forma: o petróleo é diluído em concentrações diferentes de n-heptano, aguarda-se o fenômeno de agregação dos asfaltenos e realizam-se leituras em comprimento de onda fixo. Determina-se a região de precipitação correspondente à faixa de diluição em que a curva de absorbância versus diluição apresenta um mínimo. A partir deste ponto, realizam-se diluições mais próximas desta concentração, obtendo-se outra curva como no caso anterior. O mínimo de absorbância é identificado como ponto de precipitação dos asfaltenos.

As principais dificuldades destas técnicas estão em dois fatores: a determinação em óleos viscosos (mínimo bem definido) nas curvas de titulação por NIR, identificando apenas o efeito da diluição da amostra e não da precipitação dos asfaltenos; e, além disso, por meio destas técnicas, não é considerada a inferência dos particulados presentes nos petróleos devido a sua origem (rocha reservatório).

Por microscopia óptica, o ponto de precipitação dos asfaltenos é obtido através da adição de n-heptano ao óleo, até que seja observada a formação de partículas de asfaltenos. A cada adição de solvente, a amostra é colocada em placa de Pettri e observada no microscópio (lâmina). $\mathrm{O}$ ensaio é repetido até atingir o ponto de precipitação dos asfaltenos. O final do ensaio é identificado como a diluição correspondente ao total de n- heptano adicionado no ponto anterior à ocorrência de precipitação. Este tipo de análise está fortemente associado à interpretação que induz a não repetibilidade do teste.

Através da análise de espectroscopia de varredura óptica, pode-se observar o aumento contínuo da agregação dos asfaltenos conduzido no inicio da sua floculação, pois o analisador possui dois detectores sincronizados que detectam a reflectância difusa $\left(\theta=135^{\circ}\right)$ e a transmitância $\left(\theta=0^{\circ}\right)$, em função da fração volumétrica das partículas $(\phi)$. Em regime diluído $\left(\phi<\phi_{\text {crítico }}\right)$, a transmitância decrescerá em função desta fração volumétrica e, por sua vez, se o meio estiver na condição máxima de empacotamento, uma série de fenômenos de interferên- 
cia de luz ocorrerá e o sinal retroespalhado indicará um acréscimo. Portanto, a turbidimetria dinâmica de varredura (Turbiscan) permite avaliar tanto a cinética de agregação dos asfaltenos como a presença de particulados que interferem nesse fenômeno.

\section{PARTE EXPERIMENTAL}

O princípio da técnica de espectrofotometria de varredura óptica (Turbiscan) fundamenta-se nos modelos de refletância difusa. Existem várias teorias que definem o espalhamento de luz e a utilização delas é diretamente proporcional à relação entre o comprimento de onda do feixe e o tamanho da partícula.

Os parâmetros de medidas de espalhamento através da técnica Turbiscan $^{\mathrm{TM}}$ são os definidos pela teoria Lorenz-Mie, que descreve a medida de tamanho de partícula por esferas homogêneas de tamanho arbitrário. Para partículas não esféricas, a teoria Mie considera o diâmetro esférico equivalente por volume-peso. Essa teoria não tem limitação quanto ao tamanho de partícula a ser medido. Assim sendo, atualmente, essa teoria é a mais rigorosa, gerando resultados bem próximos da realidade. A Equação 2 define a relação de transporte do fóton $\left(\lambda^{*}\right)$ no meio a ser analisado.

$$
\lambda^{*}(d, \phi)=\frac{2 d}{3 \phi(1-g) Q_{s}}
$$

onde: d - diâmetro médio da partícula; $\phi$ - fração volumétrica da fase dispersa; Q e g - parâmetros óticos obtidos através da teoria Mie.

A análise por dispersão óptica, aplicada no equipamento espectrofotômetro de varredura óptica (Turbiscan) está fundamentada na aplicação de uma fonte de luz ( $\lambda=850 \mathrm{~nm}$ ou $0,85 \mu \mathrm{m})$ que atravessa a cubeta cilíndrica contendo a amostra e dois sensores medem a quantidade de luz transmitida - transmission $(\mathrm{T})$ - que, por sua vez, representa a clarificação e a quantidade de luz espalhada - backscattering (E) - que representa a sedimentação, a $135^{\circ}$. A fonte de luz movimenta-se verticalmente, varrendo a cubeta e medindo a transmissão e o espalhamento em função da posição do tubo (Figura 1). O número de separabilidade é calculado pelo software do equipamento.

Para realização desse estudo, foram analisados dois petróleos da costa oeste africana, nomeados de A e C, e quatro amostras de petróleos oriundas das bacias produtoras brasileiras, denominadas de D, E, F e H. As caracterizações destes petróleos estão descritas nas Tabelas 1 e 2 . O critério adotado para escolha dos petróleos pe-

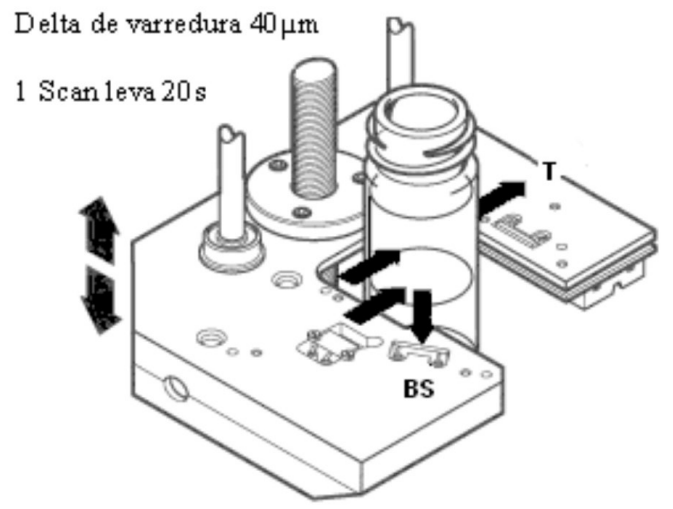

Figura 1. Ilustração do conceito do Turbiscan ${ }^{\circledast}$. Modificada do manual do equipamento modelo Turbiscan MA2000 Heavy Fuel

sados foi através da variação do índice Gaestel (Ic) (Equação 3). Os petróleos leves foram escolhidos levando-se em conta a variação do grau API (Tabela 1), pois, quanto maior o grau de saturação de um petróleo leve, maior o seu grau API.

$$
\text { Ic }=\frac{\text { Asfatenos }+ \text { Resinas }}{\text { Saturados }+ \text { Aromáticos }}
$$

O índice de instabilidade coloidal, C.I.I. - Colloidal Instability Index é a expressão dada pela Equação 4, que estabelece, em termos de classes, a instabilidade do petróleo em seus solventes. Menores teores de resinas favorecem a formação de precipitados volumosos poucos solvatados e com fraca superfície ativa. ${ }^{5,6}$

$$
\mathrm{CII}=\frac{\text { Saturados }+ \text { Asfaltenos }}{\text { Resinas }+ \text { Aromáticos }}
$$

Foram preparadas misturas destes petróleos (Tabela 3), com o objetivo de se avaliar o efeito da agregação dos asfaltenos, através dos ensaios no turbiscan. As misturas foram preparadas considerando que a porcentagem de petróleos leves foi igual ou inferior a $30 \%$.

Para a obtenção dos dados experimentais, os petróleos e suas misturas foram analisados no espectrofotômetro de varredura óptica fabricado pela Formalation ${ }^{\circledR}$, modelo Turbiscan MA 2000 Heavy Fuel. Os dados obtidos foram tratados pelo software do próprio equipamento (TurbiSoft ${ }^{\circledast}$ Version 1.2.1).

Tabela 1. Composição e propriedades dos petróleos da costa oeste africana

\begin{tabular}{cccccc}
\hline Petróleos & Grau API & Asfaltenos $^{1}(\% \mathrm{~mm})$ & Parafinas $^{2}(\%$ vol $)$ & Aromáticos $^{2}(\%$ vol $)$ & Naftênicos $^{2}\left(\% v_{0}\right)$ \\
\hline A & 34,50 & $<0,5$ & 38,27 & 19,72 & 41,93 \\
C & 41,90 & 0,10 & 42,99 & 16,88 & 40,11 \\
\hline
\end{tabular}

${ }^{1}$ Método IP143 para determinação de asfaltenos. ${ }^{12} 2$ Análise por cromatografia gasosa -PNA. ${ }^{13}$

Tabela 2. Composição e propriedades dos petróleos brasileiros

\begin{tabular}{cccccccc}
\hline Petróleos & Grau API & Asfaltenos $^{1}(\% \mathrm{~mm})$ & Saturados $^{3}(\% \mathrm{~mm})$ & Aromáticos $^{3}(\% \mathrm{~mm})$ & $\operatorname{Resinas}^{3}(\% \mathrm{~mm})$ & Ic & $\mathrm{CII}$ \\
\hline D & 20,50 & 2,92 & 33,80 & 28,50 & 34,90 & 0,61 & 0,58 \\
E & 24,90 & 2,57 & 47,20 & 30,40 & 19,83 & 0,28 & 0,99 \\
F & 13,20 & 7,30 & 35,70 & 24,60 & 32,40 & 0,66 & 0,75 \\
G & 20,00 & 1,51 & 40,40 & 35,10 & 23,00 & 0,32 & 0,72 \\
H & 27,00 & 1,57 & 54,20 & 27,70 & 16,60 & 0,22 & 1,26 \\
\hline
\end{tabular}

${ }^{3}$ Análise cromatográfica SARA por TLC - FID. ${ }^{14}$ 
Tabela 3. Misturas de petróleos utilizadas para avaliação do fenômeno de agregação

\begin{tabular}{ccccc}
\hline Amostra & \multicolumn{1}{c}{ Composição $(\% \mathrm{~m} / \mathrm{m})$} & Mistura $(\% \mathrm{~m} / \mathrm{m})$ \\
\hline $70 \mathrm{H} 28 \mathrm{~A} 2 \mathrm{~F}$ & $70 \% \mathrm{H}$ & $28 \% \mathrm{~A}$ & $2 \% \mathrm{~F}$ & \\
$70 \mathrm{G} 28 \mathrm{~A} 2 \mathrm{D}$ & $70 \% \mathrm{G}$ & $28 \% \mathrm{~A}$ & $2 \% \mathrm{D}$ & \\
$70 \mathrm{G} 28 \mathrm{C} 2 \mathrm{D}$ & $70 \% \mathrm{G}$ & $28 \% \mathrm{C}$ & $2 \% \mathrm{D}$ & \\
$70 \mathrm{H} 28 \mathrm{C} 2 \mathrm{~F}$ & $70 \% \mathrm{H}$ & $28 \% \mathrm{C}$ & $2 \% \mathrm{~F}$ & \\
$70(70 \mathrm{H} 28 \mathrm{~A} 2 \mathrm{~F}) 30 \mathrm{E}$ & & & $70 \%(70 \mathrm{H} 28 \mathrm{~A} 2 \mathrm{~F})$ & $30 \%(\mathrm{E})$ \\
$70(70 \mathrm{H} 28 \mathrm{C} 2 \mathrm{~F}) 30 \mathrm{E}$ & & & $70 \%(70 \mathrm{H} 28 \mathrm{C} 2 \mathrm{~F})$ & $30 \%(\mathrm{E})$ \\
$70(70 \mathrm{G} 28 \mathrm{~A} 2 \mathrm{D}) 30 \mathrm{E}$ & & & $70 \%(70 \mathrm{G} 28 \mathrm{~A} 2 \mathrm{D})$ & $30 \%(\mathrm{E})$ \\
$70(70 \mathrm{G} 28 \mathrm{C} 2 \mathrm{D}) 30 \mathrm{E}$ & & & $70 \%(70 \mathrm{G} 28 \mathrm{C} 2 \mathrm{D})$ & $30 \%(\mathrm{E})$ \\
\hline
\end{tabular}

As metodologias empregadas na preparação e na análise das amostras estão descritas no método ASTM D7061-06. ${ }^{1}$ Durante os ensaios, foram utilizados solventes com, no mínimo, $99 \%$ de pureza. Foram realizadas 15 varreduras com intervalo de 1 min entre cada uma delas. Analisou-se através do software do equipamento o perfil de floculação dos asfaltenos ( $\Delta$ transmissão) e a precipitação dos particulados ( $\Delta$ espalhamento).

Para avaliar os efeitos provocados tanto pelo fenômeno de agregação dos asfaltenos como pela presença de particulados, as amostras foram agrupadas em dois conjuntos de dados, conforme apresentado na Tabela 4.

Tabela 4. Conjunto de dados para avaliação dos fenômenos de agregação dos asfaltenos e precipitação

\begin{tabular}{cc}
\hline $1^{\circ}$ conjunto & $2^{\circ}$ conjunto \\
\hline $70 \mathrm{H} 28 \mathrm{~A} 2 \mathrm{~F}$ & $70 \mathrm{G} 28 \mathrm{~A} 2 \mathrm{D}$ \\
$70 \mathrm{H} 28 \mathrm{C} 2 \mathrm{~F}$ & $70 \mathrm{G} 28 \mathrm{C} 2 \mathrm{D}$ \\
$70(70 \mathrm{H} 28 \mathrm{~A} 2 \mathrm{~F}) 30 \mathrm{E}$ & $70(70 \mathrm{G} 28 \mathrm{~A} 2 \mathrm{D}) 30 \mathrm{E}$ \\
$70(70 \mathrm{H} 28 \mathrm{C} 2 \mathrm{~F}) 30 \mathrm{E}$ & $70(70 \mathrm{G} 28 \mathrm{C} 2 \mathrm{D}) 30 \mathrm{E}$ \\
\hline
\end{tabular}

As misturas foram preparadas considerando que a porcentagem de petróleos leves foi igual ou inferior a $30 \%$. As características da composição, principalmente o ${ }^{\circ} \mathrm{API}$ e o IC dos petróleos médios e pesados (Tabelas 1 e 2), também foram levadas em conta durante a preparação das amostras.

\section{RESULTADOS E DISCUSSÃO}

As misturas de petróleos e os petróleos puros do primeiro conjunto de dados foram submetidas à técnica de varredura óptica. $\mathrm{O}$ gráfico do delta de transmissão referente à amostra $\mathrm{H}$ é mostrado na Figura 2 e o gráfico de sedimentação e clarificação em função do tempo é mostrado na Figura 3. O número de separabilidade, calculado através da metodologia descrita na norma ASTM D7061-06, ${ }^{1}$ é mostrado na Tabela 5.

A partir dos resultados obtidos, foi possível construir o gráfico de barra mostrando o $\Delta$ transmissão (\%) e o $\Delta$ espalhamento(\%) para cada amostra (Figura 4) com o objetivo de avaliar a relação entre os fenômenos de coalescência e de precipitação obtidos através da cinética.

Foram adicionados ao petróleo $\mathrm{H}$ um petróleo leve, A (28\%), e um petróleo pesado, F (2\%), que deveriam aumentar a camada de transmissão, já que a quantidade de petróleo leve foi muito superior à quantidade de petróleo pesado adicionada. $\mathrm{O}$ efeito inverso ao esperado foi observado, quando a camada de transmissão diminuiu e a camada de precipitação (espalhamento) aumentou. Adicionando

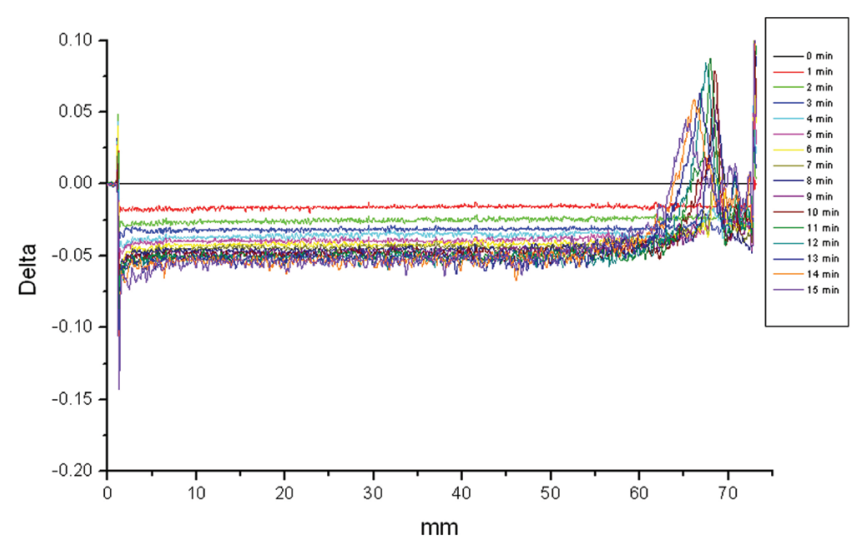

Figura 2. Gráfico do delta de transmissão referente à amostra $H$

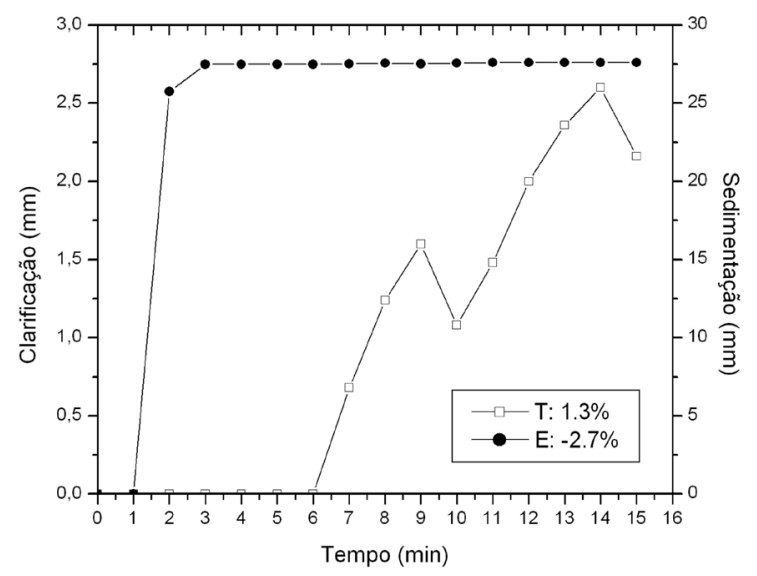

Figura 3. Gráfico de sedimentação e clarificação em função do tempo referente à amostra $H$

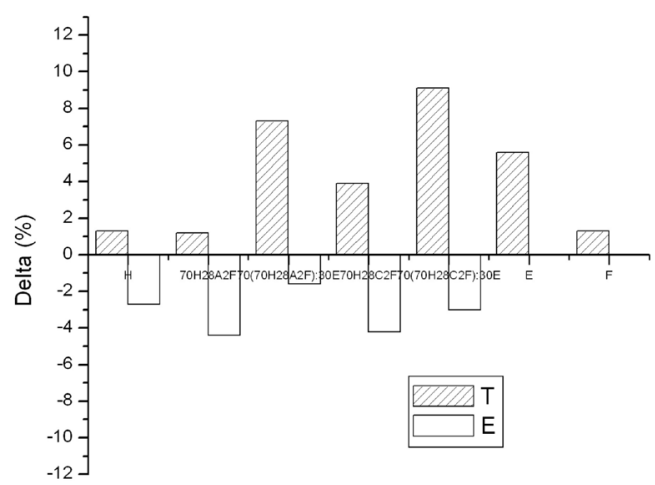

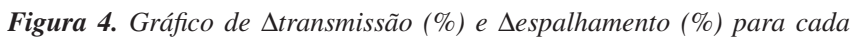
amostra do primeiro conjunto de dados

Tabela 5. Resultados obtidos para o primeiro conjunto de amostras analisadas através da técnica de varredura óptica (Turbiscan)

\begin{tabular}{cccc}
\hline $\begin{array}{c}\text { Amostras do } \\
1^{\circ} \text { conjunto de dados }\end{array}$ & $\begin{array}{c}\text { Número de } \\
\text { separabilidade }\end{array}$ & $\begin{array}{c}\Delta \text { transmissão } \\
(\%)\end{array}$ & $\begin{array}{c}\Delta \text { espalhamento } \\
(\%)\end{array}$ \\
\hline $70 \mathrm{H} 28 \mathrm{~A} 2 \mathrm{~F}$ & 1,67 & 2,80 & 8,70 \\
$70 \mathrm{H} 28 \mathrm{C} 2 \mathrm{~F}$ & 1,77 & 1,30 & 13,90 \\
$70(70 \mathrm{H} 28 \mathrm{~A} 2 \mathrm{~F}) 30 \mathrm{E}$ & 4,70 & 7,30 & 1,60 \\
$70(70 \mathrm{H} 28 \mathrm{C} 2 \mathrm{~F}) 30 \mathrm{E}$ & 7,26 & 9,10 & 3,50 \\
$\mathrm{H}$ & 1,48 & 1,40 & 5,30 \\
$\mathrm{~F}$ & 0,74 & 1,10 & 0 \\
$\mathrm{E}$ & 4,84 & 7,90 & 0 \\
\hline
\end{tabular}


o petróleo leve C (28\%) e o petróleo pesado $\mathrm{F}(2 \%)$, observou-se que as camadas de precipitação e de floculação aumentaram. Com relação à camada de transmitância, a composição do petróleo leve $\mathrm{C}$ altera a cinética de floculação. Estes fenômenos podem estar relacionados a dois fatores: primeiro, ao fato de que o decréscimo da relação resinas/asfaltenos favorece uma rápida precipitação dos asfaltenos, como descrito por Mc Lean. ${ }^{9}$ Em segundo lugar, a presença de particulados favorece o retardo da floculação dos asfaltenos, conforme foi observado nos estudos conduzidos por Dudášová. ${ }^{15}$ Estes autores observaram que, dependendo da concentração de particulados e de sua característica (tamanho das partículas), durante os 15 primeiros min de ensaio, estes revestem os asfaltenos, tendo efeito dominante na cinética. Menores teores de resinas favorecem a formação de precipitados volumosos poucos solvatados e com fraca superfície ativa. O retardo na floculação dos asfaltenos também pode ser verificado devido à presença de particulados na amostra $(\mathrm{H})$.

Foi adicionado o petróleo $\mathrm{E}$ nas duas misturas anteriores (70H28A2F e 70H28C2F) com o objetivo de avaliar a possibilidade de reversão do fenômeno de agregação. Este petróleo possui uma composição que favorece o fenômeno de desagregação, devido à presença de menores teores de resinas, como pode ser observado na Tabela 2. Verificou-se que a adição deste petróleo possibilitou o aumento da camada de floculação e diminuição da camada de precipitação em ambos os casos. A presença de menores teores de resinas no petróleo E garantiu a ocorrência do fenômeno de floculação em detrimento da presença de particulados. Menores teores de resinas favorecem a formação de precipitados volumosos poucos solvatados e com fraca superfície ativa.

Estes ensaios mostram que a composição dos petróleos serve como parâmetro de previsão do fenômeno de agregação, salvo a presença de particulados, comprovando a necessidade de se avaliar os dois fenômenos simultaneamente.

O conjunto de dados apresentado na Tabela 2 contém petróleos com menores teores de asfaltenos. O gráfico do delta de transmissão referente à amostra G é mostrado na Figura 5 e o gráfico de sedimentação e clarificação em função do tempo é mostrado na Figura 6. O número de separabilidade, calculado através da metodologia descrita na norma ASTM D7061-06, ${ }^{1}$ é mostrado na Tabela 6. O gráfico de barras barra mostrando o $\Delta$ transmissão (\%) e o $\Delta$ espalhamento(\%) para cada amostra do segundo conjunto de dados é mostrado na Figura 7.

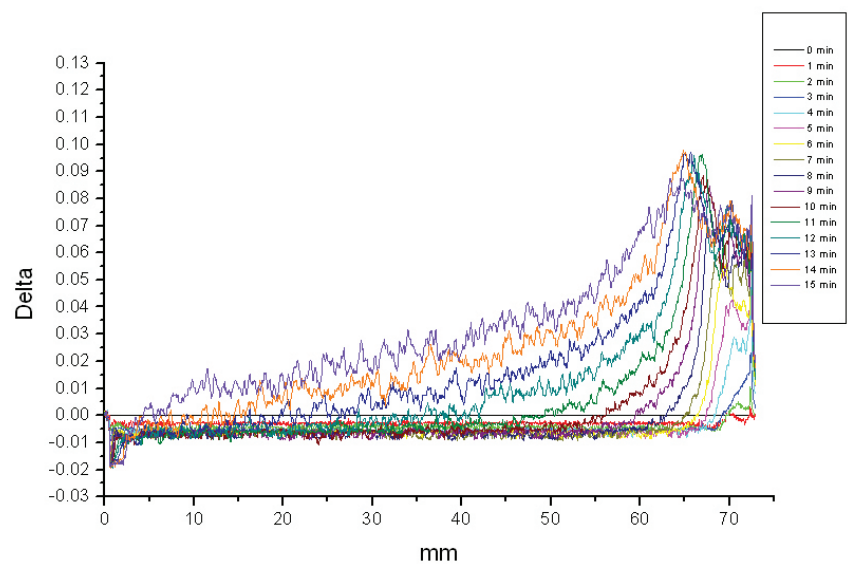

Figura 5. Gráfico do delta de transmissão referente à amostra $G$

Foi adicionado ao petróleo G, $28 \%$ do petróleo leve A e 2\% de um petróleo médio $\mathrm{D}$, que deveriam aumentar a camada de transmissão. Foi observado que a camada de transmissão aumentou, o que ocorreu também com a camada de precipitação. Adicionando-se $28 \%$ do petróleo leve $\mathrm{C}$ e $2 \%$ do petróleo D ao petróleo G, observou-se

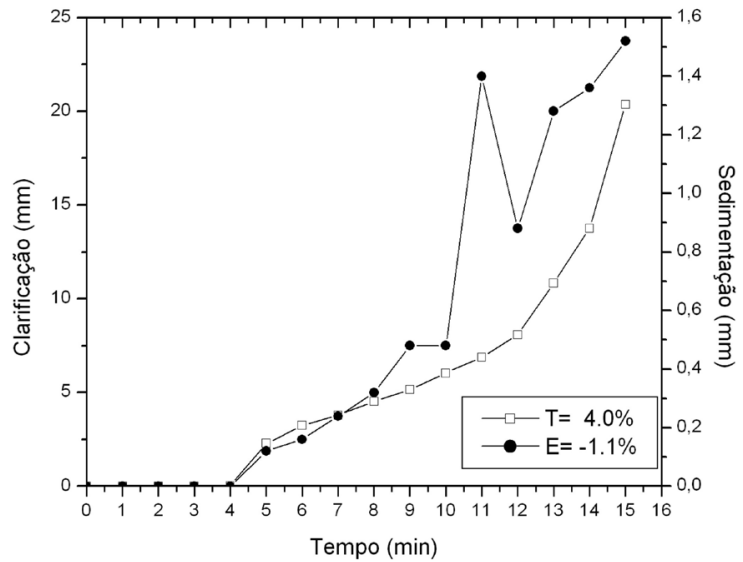

Figura 6. Gráfico de sedimentação e clarificação em função do tempo referente à amostra $G$

Tabela 6. Resultados obtidos para o segundo conjunto de amostras analisadas através da técnica de varredura óptica (Turbiscan)

\begin{tabular}{cccc}
\hline $\begin{array}{c}\text { Amostra do } 2^{\circ} \\
\text { conjunto de dados }\end{array}$ & $\begin{array}{c}\text { Número de } \\
\text { separabilidade }\end{array}$ & $\begin{array}{c}\Delta \text { transmissão } \\
(\%)\end{array}$ & $\begin{array}{c}\Delta \text { espalhamento } \\
(\%)\end{array}$ \\
\hline 70G28A2D & 1,17 & 4,80 & 3,30 \\
70G28C2D & 2,45 & 6,60 & 3,30 \\
70(70G28A2D)30E & 4,60 & 6,40 & 0,40 \\
70(70G28C2D)30E & 4,62 & 7,10 & 0,60 \\
G & 0,92 & 4,00 & 1,00 \\
D & 1,21 & 1,90 & 0 \\
E & 4,84 & 7,90 & 0 \\
\hline
\end{tabular}

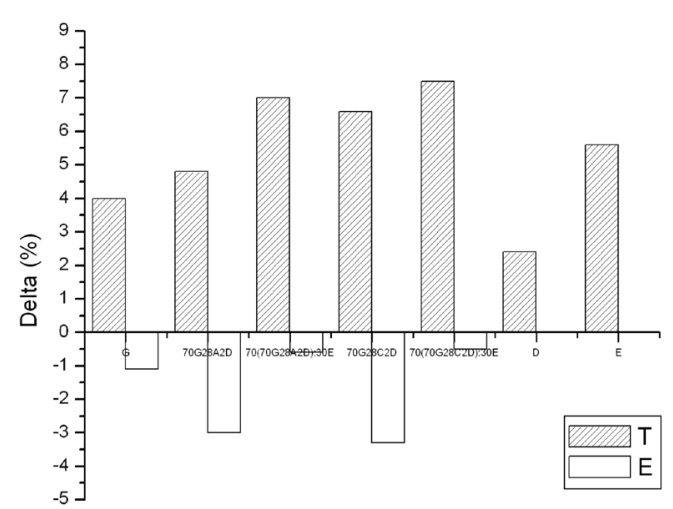

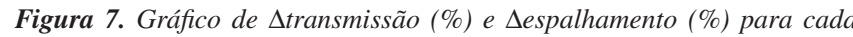
amostra do segundo conjunto de dados

o mesmo comportamento, as camadas de transmisão e precipitação aumentaram.

Como pode ser observado na Figura 7, a adição de $30 \%$ do petróleo $\mathrm{E}$ (baixos teores de resinas) às misturas anteriores promoveu uma maior floculação dos asfaltenos e a diminuição da camada de precipitação. Isto ocorre devido aos asfaltenos estarem revestidos pelos particulados, tendo um efeito dominante na suspensão. Ao se adicionar o petróleo E, este revestimento é quebrado, ocorrendo o fenômeno de clarificação e não havendo retardo na floculação.

Observa-se que as amostras do segundo conjunto de dados estão menos solvatadas pelo solvente alifático, formando uma camada de precipitação menos espessa. Outro fenômeno observado foi o da relação direta entre a escolha dos petróleos com teores de saturados ao processo de floculação. Por exemplo, a amostra contendo um 
teor mais elevado de saturados, amostra C, apresentou valores mais elevados na camada de floculação.

A presença de particulados que favorecem o retardo da floculação dos asfaltenos também foi observada, mas com menor intensidade, devido à menor concentração de asfaltenos deste conjunto de amostras. Este comportamento pode ser observado na Figura 7, em que temos o delta de transmitância das amostras G e D. Estas amostras têm em sua composição química diferenças significativas (Tabela 2) principalmente em relação ao índice de instabilidade coloidal, C.I.I. (Equação 4). C.I.I., Colloidal Instability Index, é a expressão dada para estabelecer, em termos de classe, a instabilidade do petróleo em seus solventes. Menores teores de resinas favorecem a formação de precipitados volumosos poucos solvatados e com fraca superfície ativa.

O retardo na floculação dos asfaltenos também pode ser verificado devido à presença de particulados na amostra G. ${ }^{9}$ Outro aspecto a ser ressaltado é que as resinas têm papel importante na estabilização dos asfaltenos em solução, dispersando os asfaltenos e impedindo sua separação. Quando calculamos o índice Gaestel (Ic), que estabelece a capacidade de dispersão dos maltenos frente aos asfaltenos, verificamos que a amostra $\mathrm{G}$ tem seu valor calculado de 0,32 e a amostra $\mathrm{D}$, de 0,61 ; portanto, quando temos um acréscimo no valor do índice Gaestel, ocorre a diminuição da estabilidade coloidal. ${ }^{4}$

A floculação também foi favorecida pela relação da composição da amostra E que tem seu valor calculado do índice Gaestel de 0,29. Portanto, ocorre o aumento da estabilidade coloidal favorecida pelo poder de solubilização das resinas, que é afetado pelo grau de aromaticidade das moléculas.

\section{CONCLUSÕES}

A avaliação da composição (SARA) dos petróleos puros serve como parâmetro para avaliação do comportamento da cinética de precipitação e de floculação. Foram avaliados modelos para tratar da cinética do processo de agregação dos asfaltenos e um estudo sobre o processo de floculação através de um novo método.

Somente a avaliação dos resultados obtidos através da norma ASTM não é suficiente para a avaliação do comportamento das misturas durante o processamento dos óleos, pois avalia apenas os resultados obtidos no detector de transmitância. Tanto nas análises de cinética do processo de agregação (floculação) como através da análise da formação de partículas (clarificação), foi possível identificar e comparar o comportamento das amostras de acordo com a variação da sua composição. Abre-se uma nova oportunidade de metodologia de análise para avaliação de problemas de compatibilidade de petróleos e identificação de amostras que podem formar emulsões estáveis durante o processamento.
Através dos resultados, verifica-se a importância das resinas na estabilidade dos asfaltenos em solução, contribuindo para a dispersão dos asfaltenos e impedindo a sua separação. A aplicação do índice Gaestel, que estabelece a capacidade de dispersão dos maltenos aos asfaltenos, foi evidenciada nas misturas contendo petróleos com valores com índice Gastel mais elevados. Esta situação evidencia a diminuição da estabilidade coloidal aumentado, portanto, a camada de floculação.

\section{AGRADECIMENTOS}

À PETROBRAS e à FAPESP pelo apoio financeiro.

\section{REFERÊNCIAS}

1. ASTM D 7061 - 06, Standard Test Method for Measuring n-Heptane Induced Phase Separation of Asphaltene-Containing Heavy Fuel Oils as Separability Number by an Optical Scanning Device.

2. Janardhan, A. S.; Mansoori, G. A.; J. Petroleum Science Engineering 1993, 9, 17.

3. Leon, O.; Rogel, E.; Espidel, J.; Torres, G.; Energy Fuels 2000, 14, 6.

4. Oyekunle, L. O.; Oil \& Gas Science and Technology-Rev. IFP 2006, 61.

5. Asomaning, S.; Watkinson, A. P.; Heat Transfer Engineering 2000, 16, 21.

6. Srinivasan, M.; Watkinson, A. P.; ECI Conference on Heat Exchanger Fouling and Cleaning: Fundamentals and Applications, Santa Fe, USA, 2003.

7. http://www.aiche-chicago.org/symposium06/wiehe.pdf, acessada em Janeiro 2011.

8. Bennett, C. A.; Kistler, R. S.; Nangia, K.; Al-Ghawas W.; Al-Hajji, N.; Al-Jemaz A.; Proceedings of 7th International Conference on Heat Exchanger Fouling and Cleaning - Challenges and Opportunities, Tomar, Portugal, 2007.

9. McLean, J. D.; Kilpatrick, P. K.; J. Colloid Interface Sci. 1997, 196, 23.

10. Wiehe, I. A.; Kennedy, R. J.; Energy Fuels 2000, 14, 56.

11. Zílio, E. V.; Santos, M. F. P.; Camacho, C. F. B.; Boletim Técnico Petrobras 2003, 46, 247.

12. ASTM D6560 (IP 143), Determination of Asphaltenes (Heptane Insolubles) in Crude Petroleum and Petroleum Products.

13. ASTM D 5443 - 04, Paraffin, Naphthene, and Aromatic Hydrocarbon Type Analysis in Petroleum Distillates through $200{ }^{\circ} \mathrm{C}$ by Multi-Dimensional Gas Chromatography.

14. Jiang, C.; Larter, S. R.; Noke, K. J.; Snowdon, L. R.; Org. Geochem. 2008, 39, 1210 .

15. Dudášová, D.; Flåten, G. R.; Sjöblom, J.; Øye, G.; Colloids Surf., A 2009, 335, 62 